\title{
COMPARISON OF COLOUR REPRODUCTION BY PENTAX K10D DIGITAL CAMERA EMPLOYING POLYNOMIAL MODELS AND ICC BASED COLOUR MANAGEMENT TOOLS
}

\author{
Ondrej Panák (D), Natálie Kailová (iD, Markéta Držková \\ University of Pardubice, Faculty of Chemical-Technology \\ Department of Graphic Arts and Photophysics, Pardubice, Czech Republic
}

\begin{abstract}
This paper deals with the characterization of a Pentax $K 10$ digital camera in order to be used in colorimetric measurements in a custom built setup employing a LED light source. The experiment focuses on colour characterization using several polynomial transformation models in comparison to ICC based colour characterisation. Altogether 5 polynomial models are applied and evaluated by capturing set of uniform colour patches. Preliminary results indicate, that the complexity of the model does not markedly improve the prediction of CIELAB values.
\end{abstract}

Key words: digital camera, calibration, characterisation, polynomial models, colour prediction

\section{INTRODUCTION}

Digital $R G B$ cameras can be utilised in applications mapping dynamic colour change of thermochromics surfaces (Abdullah et al, 2010; Smith et al, 2001; Farina et al, 1994; Vejrazka et al, 2007; Cukurel et al, 2012; Bourque et al, 2015; Panák et al, 2018). In such cases, the most important information is the information about the magnitude of colour change in dependence of temperature. The $R G B$ signal recorded by the camera has to be transformed into colorimetric representation. There are several methods that could be employed: spectral characterisation of camera sensors (Cheung et al, 2005; Sole et al, 2016; Jiang et al, 2013), neural networks (Cheung et al, 2004) and polynomial modelling (Cheung et al, 2004; Westland et al, 2012; Bianco et al, 2009; Johnson, 1996; Hong et al, 2001; Finlayson et al, 2015).

Polynomial modelling requires uniform illumination and stable conditions in order to obtain reproducible results (Cheung et al, 2004; Westland et al, 2012; Bianco et al, 2009). A set of about 60 colour patches applied as training set seems to be enough to obtain good colorimetric prediction (Hong et al, 2001). The polynomial modelling transforms linearized $R G B$ values into CIEXYZ colorimetric values. An example of polynomial model is shown in Equation 1.

$$
\begin{aligned}
& X=a_{1,1} R+a_{1,2} G+a_{1,3} B+a_{1,4} R G+a_{1,5} R B+a_{1,6} G B+a_{1,7} R^{2}+a_{1,8} G^{2}+a_{1,9} B^{2}+a_{1,10} \\
& Y=a_{2,1} R+a_{2,2} G+a_{2,3} B+a_{2,4} R G+a_{2,5} R B+a_{2,6} G B+a_{2,7} R^{2}+a_{2,8} G^{2}+a_{2,9} B^{2}+a_{2,10} \\
& Z=a_{3,1} R+a_{3,2} G+a_{3,3} B+a_{3,4} R G+a_{3,5} R B+a_{3,6} G B+a_{3,7} R^{2}+a_{3,8} G^{2}+a_{3,9} B^{2}+a_{3,10}
\end{aligned}
$$

Polynomial model can be more or less complex. Polynomial models of second degree seem to be enough good to predict colorimetric data from RGB values (Hong et al, 2001). The polynomial transformation can be expressed in matrix form by Equation 2 (Cheung et al, 2004; Westland et al, 2012; Bianco et al, 2009).

$\mathbf{T}=\mathbf{D} \quad \mathbf{A}$

Matrix T represents $n \times 3$ values of $X Y Z$ and matrix $D$ is matrix of $n \times m$ extended values of $R G B$. The $n$ represents the size of the set, e.g the number of colour patches or pixels. From Equation 1 the $m$ is 10 $\left(R, G, B, R G, R B, G B, R^{2}, G^{2}, B^{2}, 1\right)$. Matrix A represents coefficients of selected polynomial model. In order to obtain coefficients of the model, colour patches of some training set have to be recorded. Then the matrix $\mathrm{A}$ can be found by Equation 3.

$$
\mathbf{A}=\mathbf{D}^{+} \quad \mathbf{T}
$$

Matrix T represent known set of CIEXYZ values of training colour patches. Matrix $\mathrm{D}^{+}$is a pseudoinverse (Cheung et al, 2004; Westland et al, 2012; Bianco et al, 2009) matrix, determined from known set of RGB values of training colour patches. When coefficients of the polynomial model are known, any $R G B$ combination can be transformed into CIEXYZ combination using Equation 1. Extension of the polynomial model can reduce the error for about 50 \% (Bianco et al, 2009; Johnson, 1996; Hong et al, 2001; Finlayson et al, 2015), but only in case of stable capturing conditions (Finlayson et al, 2015). 
The aim of the paper is to compare two approaches of transforming $R G B$ data into CIELAB data in the setup we have applied in our previous study (Panák et al, 2018). The first approach is ICC based transformation in Adobe Photoshop. The second approach applies custom built algorithms of different polynomial models.

\section{EXPERIMENTAL}

\subsection{Devices}

Raw digital RGB image data were captured by Pentax K10D camera with SMC Pentax-DA 1:4(22) 16-45 mm ED-AL lens. A Falcon Eyes' ring LED source DVR-630DVC with diffusor was utilized as a flat diffusive illumination with CRI value equal to 94 , as specified by the producer (Falconeyes, 2018). Colorimetric parameters of colour patches were measured by Hunterlab UltraScan Vis spectrophotometer with $\mathrm{SCl} d / 8$ geometry.

\subsection{Colour charts}

Three colour charts were applied in this study. The first one was X-Rite ColorChecker Passport Photo with 24 patches, including 6 patches of neutral colours (See Figure 1). Colorimetric data provided by the producer (Xritephoto, 2018) were applied as a reference. The second custom made Profiling colour chart contained 80 colour patches including 8 neutral colours (see Figure 2). The third Testing colour chart contained another 80 colour patches that were selected randomly and were different from colour patches in Profiling colour chart (see Figure 3). Profiling and Testing colour patches were cut out of the NCS Index 1950 sample collection (NCS Colour AB, 2017) and their reference CIEXYZ colorimetric data were captured by Hunterlab UltraScan Vis spectrophotometer (D50, $2^{\circ}$ observer). These charts were subdivided each in four parts, so the size matches approximately the size of ColorChecker Passport.

\subsection{Methods}

The digital camera was placed into the middle of a circular opening of the light source. The distance of the light source from the surface of measured sample was about $38 \mathrm{~cm}$. The space between the light source and the sample was covered by a protective skin with an internal white diffusive surface, to prevent the negative effect of the outer environment. The sensitivity of the camera was set to ISO 200 and optimal shutter speed and aperture were found and kept constant over the experiment. The focal length of the lens was adjusted so the captured colour chart took about one fourth of total area captured by the sensor.

Stability of camera output was investigated by capturing 300 images of a white substrate with diffusive coating and white balance target of ColorChecker in 7 seconds intervals. Camera Raw 7.0 module of Adobe Photoshop CS4 software was used to obtain 16 bit RGB tiff images from raw DNG file. The stability was evaluated in terms of development of RGB values over time. Capturing of the sequence started after at least 30 minutes from switching on the light source. After this time the intensity of the source is stabilised. All colour charts described in previous chapter were captured in a sequence one after each other and they were processed later.

In order to perform fairly good linearization of RGB values, first the optimal transformation of DNG file into TIFF file had to be found. This was done by preparing set of 16 bit TIFF images out of one DNG file of CholorChecker in Camera Raw 7.0 module, where values of selected parameters were set to certain value or option. These parameters were: Exposure, Blacks, Brightness, Contrast, Curves, Details, and DNG profile. All other parameters were of 0 value. A custom made DNG profile of the camera was generated by ColorChecker Passport software. Only neutral colour patches of ColourChecker were considered in the procedure. The objective was to determine coefficients of Equation 4:

$C_{\mathrm{i}}=a_{\mathrm{i}} R_{\mathrm{i}}^{b_{\mathrm{i}}}$

where $C_{\mathrm{i}}$ is value of $X, Y$, or $Z$ and $R_{\mathrm{i}}$ is value of $R, G$, and $B$ respectively. The setup of Camera Raw 7.0 resulting in the best fit was considered in further processing of all other images. Coefficients of Equation 4 found for the best fit were used in linearization of $R G B$ values of all colour patches. 


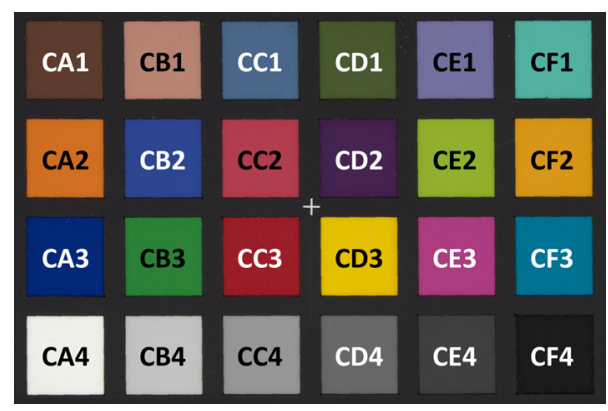

Figure 1: ColorChecker Pasport

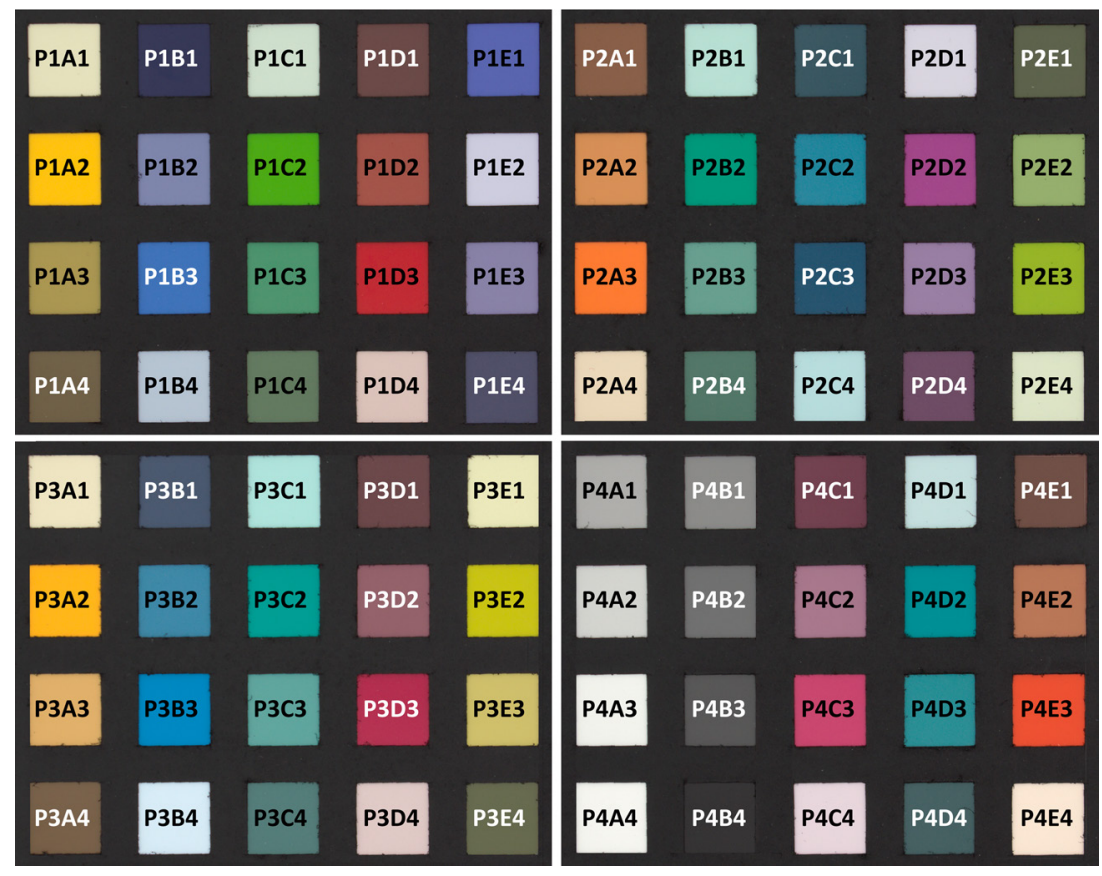

Figure 2: Profiling colour chart

\begin{tabular}{|c|c|c|c|c|c|c|c|c|c|}
\hline T1A1 & T1B1 & T1C1 & T1D1 & T1E1 & T2A1 & T2B1 & T2C1 & T2D1 & T2E1 \\
\hline T1A2 & T1B2 & T1C2 & T1D2 & T1E2 & T2A2 & T2B2 & T2C2 & T2D2 & T2E2 \\
\hline T1A3 & T1B3 & T1C3 & T1D3 & T1E3 & T2A3 & T2B3 & $\mathrm{T} 2 \mathrm{C} 3$ & T2D3 & T2E3 \\
\hline T1A4 & T1B4 & T1C4 & T1D4 & T1E4 & T2A4 & T2B4 & T2C4 & T2D4 & T2E4 \\
\hline T3A1 & T3B1 & T3C1 & T3D1 & T3E1 & T4A1 & T4B1 & T4C1 & T4D1 & T4E1 \\
\hline T3A2 & T3B2 & T3C2 & T3D2 & T3E2 & T4A2 & T4B2 & T4C2 & T4D2 & T4E2 \\
\hline T3A3 & T3B3 & T3C3 & T3D3 & T3E3 & T4A3 & T4B3 & T4C3 & T4D3 & T4E3 \\
\hline T3A4 & T3B4 & T3C4 & T3D4 & T3E4 & T4A4 & T4B4 & T4C4 & T4D4 & T4E4 \\
\hline
\end{tabular}

Figure 3: Testing colour chart 
In the ICC based colour transformation the camera ICC profile had to be created. It was done in i1Profiler software from linearized 16 bit RGB TIFF file of ColorChecker. Created profile was assigned to all colour charts and the image was converted to CIELAB using absolute colorimetric rendering intent in Adobe Photoshop CS4. Obtained images were saved as 16 bit TIFF file. Mean CIELAB values of each colour patch were compared to reference CIELAB values in Matlab 2015 by means of $\triangle E_{00}$ applying predefined function (Westland et al, 2012).

Based on the information in (Cheung et al, 2005), all together 5 polynomial models (see Table 1) were tested using the general Equation 2. The coefficient matrix A was found by Equation 3 applying the pinv function in Matlab. In one case, the matrix $A$ was found while linearized $R G B$ values of CholorChecker were set to be the training set. In the second case, linearized $R G B$ values of Profiling colour chart were set as training set. Each polynomial model with generated coefficients was then applied to linearized $R G B$ values of all colour patches mentioned in 2.2. Linearization of $R G B$ values of all colour patches was done according to neutral colour patches of corresponding training set. Obtained theoretical colorimetric representation was compared to reference colorimetric data by means of $\Delta E_{00}$.

Table 1: List of polynomial models represented by formulation of matrix D

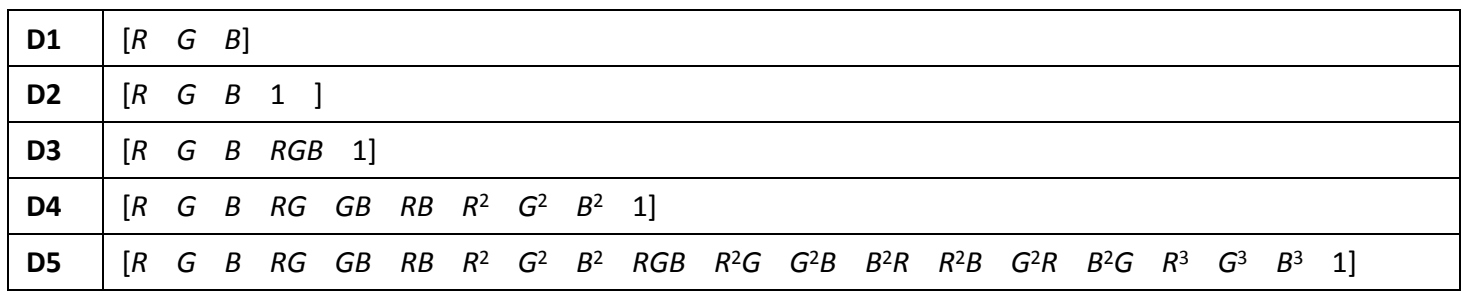

\section{RESULTS AND DISCUSSIONS}

\subsection{Stability of the camera output}

Figure 4a shows the development of $R G B$ values over time when capturing of the sequence started immediately after switching on the camera. It can be seen that the camera white balance is not kept constant. After approximately three minutes, some splitting of the magnitude id $R, G$ and $B$ values can be observed. Figure $4 \mathrm{~b}$ shows the case, when capturing started 40 minutes after switching on the camera. The white balance was kept constant over time. Therefore the capturing of all colour patches started always at least 40 minutes after the camera was switched on. Some noise in the signal intensity can be observed, most probably due to a mechanical shutter of studied camera. The evaluation of variability in CIELAB colour space of ColorChecker can be found elsewhere (Panák et al, 2018).
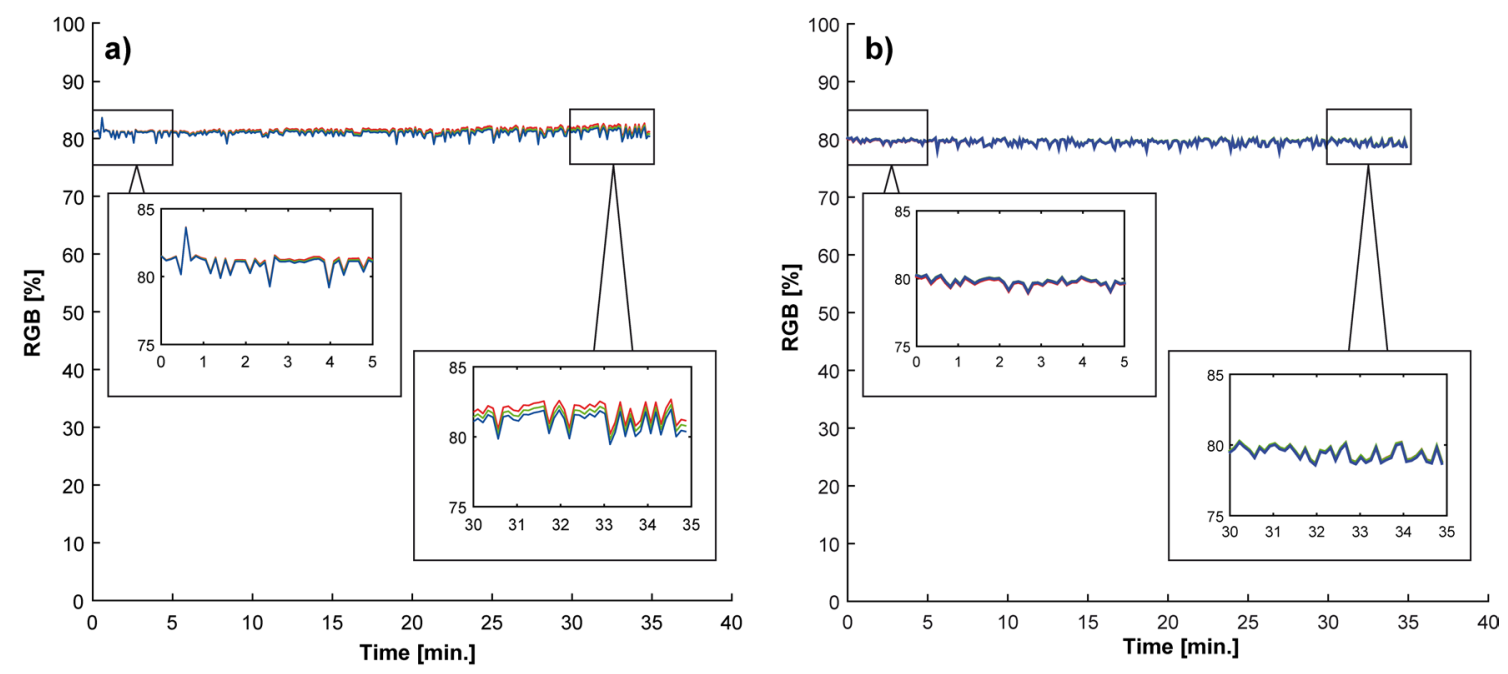

Figure 4: Camera output stability 


\subsection{DNG to TIFF transformation}

The best setup of transforming DNG file into TIFF tile in Camera Raw 7.0 was setup described in the Table 2 as setup C4. Figure 5a illustrates the fit of $X(R), Y(G)$ and $Z(B)$ dependencies according to general formula in Equation 4. Setup C13 is shown in Figure $5 b$ for comparison, where obtained data do not fit the exponential function as good as in case of C4. Change in the exposition (C3-C8) did not affect the quality of regression. Setup C4 was applied in creation of RGB TIFF files of all colour patches.

Table 2: Determination coefficients $R^{2}$ of $X(R), Y(G)$ and $Z(B)$ functions for different setups of Camera Raw 7.0.

\begin{tabular}{|c|c|c|c|c|c|c|c|c|c|c|}
\hline & 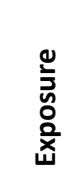 & $\begin{array}{l}\frac{\tilde{y}}{0} \\
\frac{\pi}{\infty}\end{array}$ & 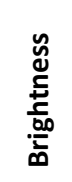 & 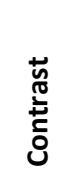 & 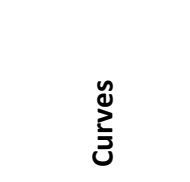 & 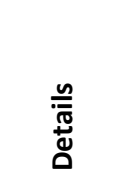 & 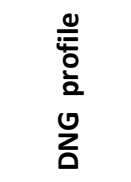 & \multicolumn{3}{|c|}{$\propto$} \\
\hline & & & & & & & & $\mathrm{R}$ & G & B \\
\hline C1 & 0,00 & 0 & +50 & +25 & linear & implicit & custom & 0.9921 & 0.9904 & 0.9912 \\
\hline C2 & 0,00 & 5 & +50 & +25 & linear & 0 & custom & 0.9911 & 0.9893 & 0.9902 \\
\hline C3 & 1,00 & 5 & 0 & 0 & linear & implicit & custom & 0.9997 & 0.9999 & 0.9998 \\
\hline C4 & 0,90 & 5 & 0 & 0 & linear & implicit & custom & 0.9999 & 0.9997 & 0.9998 \\
\hline C5 & 0,80 & 5 & 0 & 0 & linear & implicit & custom & 0.9997 & 0.9999 & 0.9998 \\
\hline C6 & 0,70 & 5 & 0 & 0 & linear & implicit & custom & 0.9997 & 0.9999 & 0.9998 \\
\hline C7 & 0,60 & 5 & 0 & 0 & linear & implicit & custom & 0.9997 & 0.9999 & 0.9998 \\
\hline C8 & 0,50 & 5 & 0 & 0 & linear & implicit & custom & 0.9997 & 0.9999 & 0.9998 \\
\hline C9 & 0,00 & 0 & 0 & 0 & linear & implicit & custom & 0.9993 & 0.9997 & 0.9995 \\
\hline C10 & 0,00 & 0 & 0 & +25 & linear & implicit & custom & 0.9996 & 0.9999 & 0.9998 \\
\hline C11 & 0,00 & 5 & 0 & +25 & linear & implicit & custom & 0.9965 & 0.9952 & 0.9957 \\
\hline C12 & 0,00 & 5 & +50 & 0 & linear & implicit & custom & 0.9912 & 0.9891 & 0.9903 \\
\hline C13 & 0,00 & 5 & +50 & +25 & linear & implicit & ACR 4.4 & 0.9912 & 0.9891 & 0.9900 \\
\hline C14 & 0,00 & 5 & +50 & +25 & middle contr. & implicit & ACR 4.4 & 0.9908 & 0.9888 & 0.9900 \\
\hline C15 & 0,00 & 5 & +50 & +25 & middle contr. & implicit & Adobe & 0.9912 & 0.9892 & 0.9904 \\
\hline C16 & 0,00 & 5 & +50 & +25 & middle contr. & implicit & custom & 0.9912 & 0.9891 & 0.9903 \\
\hline
\end{tabular}
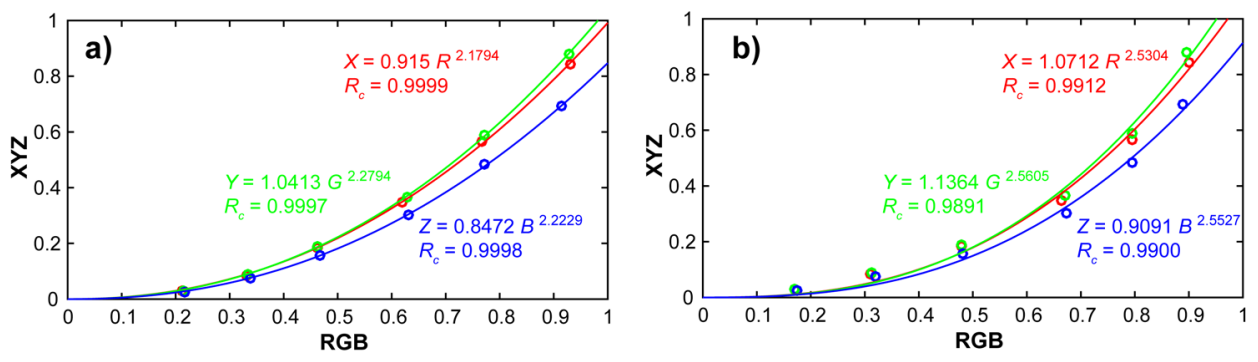

Figure 5: Regression of $X(R), Y(G)$ and $Z(B)$ dependencies for setup C4 (a) and C13 (b)

\subsection{ICC based colour transformation}

The colour difference $\Delta E_{00}$ together with difference in attributes is presented in Table 3 . As expected, the colour difference on ColorChecker is fairly low, not exceeding the value 2 (see Figure $6 a)$. In case of the Profiling and Testing colour chart the worst prediction, $\Delta E_{00}$ exceeds the value 4, is for brown and dark green colours (see Figure 6b,c). The unsatisfactory colour prediction could be affected by indirect glare effects observed during the measurement. The surface of Profiling and Testing colour patches was semi-matte, glossier when compared to patches of ColourChecker. Some variability due to stability of camera output can also have a slight influence on the magnitude of $\Delta E_{00}$. 
Table 3: Colour differences in case of ICC based RGB to CILEAB transformation

\begin{tabular}{|c|r|c|c|c|c|}
\hline & & $\boldsymbol{\Delta} \boldsymbol{E}_{\mathbf{0 0}}$ & $\boldsymbol{\Delta}$ & $\boldsymbol{\Delta}$ & $\boldsymbol{\Delta}$ \\
\hline \multirow{3}{*}{ Median } & ColorChecker & 0.71 & 0.25 & 0.39 & 0.34 \\
\cline { 2 - 6 } & Profiling & 2.35 & 1.62 & 0.73 & 0.84 \\
\cline { 2 - 6 } & Testing & 2.25 & 1.09 & 0.75 & 0.80 \\
\hline \multirow{3}{*}{ Minimum } & ColorChecker & 0.12 & 0.01 & 0.03 & 0.05 \\
\cline { 2 - 6 } & Profiling & 0.47 & 0.01 & 0.05 & 0.00 \\
\cline { 2 - 6 } & Testing & 0.68 & 0.03 & 0.01 & 0.00 \\
\hline \multirow{3}{*}{ Maximum } & ColorChecker & 1.92 & 1.80 & 0.96 & 1.31 \\
\cline { 2 - 6 } & Profiling & 4.84 & 4.76 & 2.26 & 4.10 \\
\cline { 2 - 6 } & Testing & 5.82 & 5.05 & 2.55 & 4.12 \\
\hline
\end{tabular}
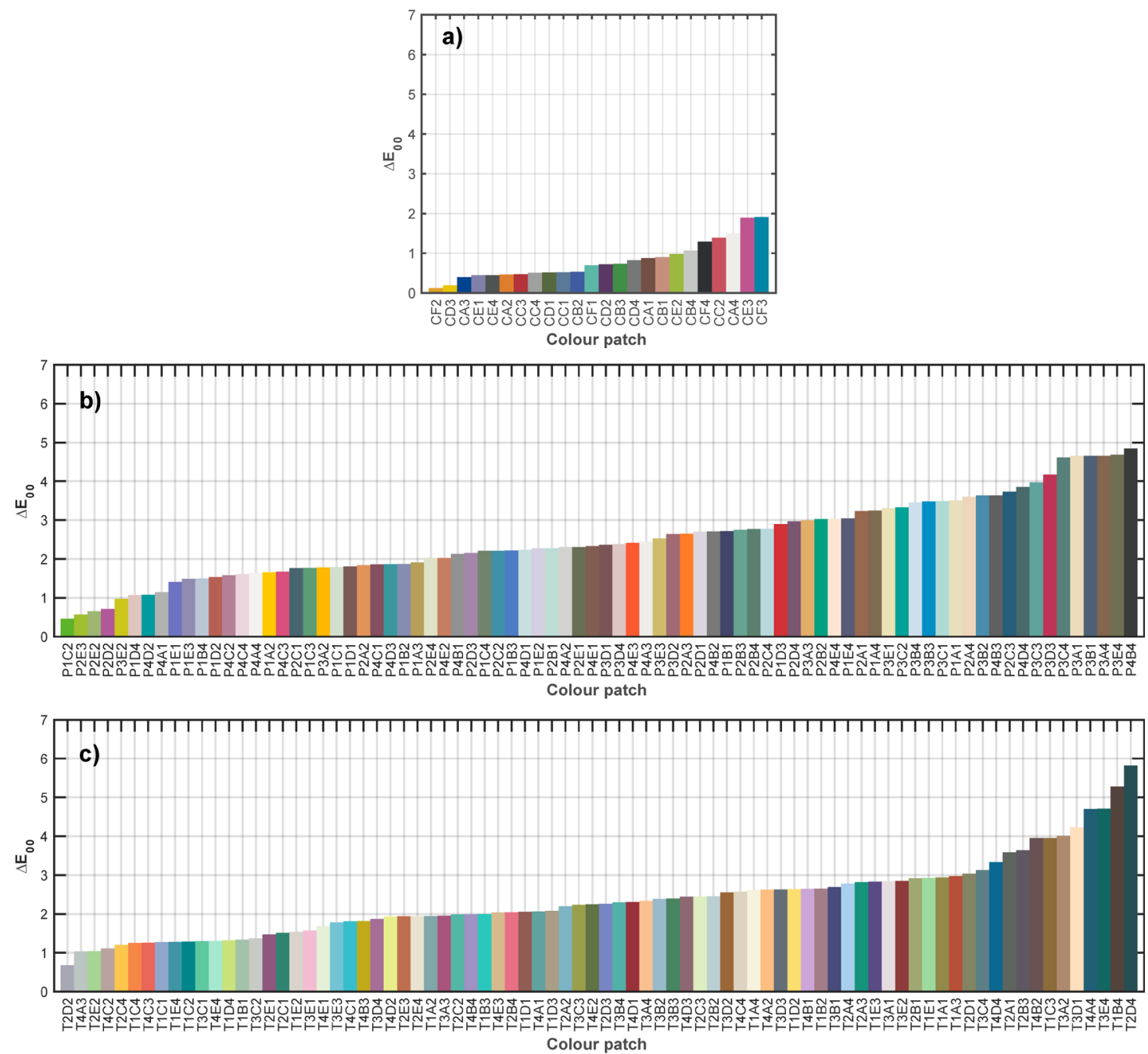

Figure 6: Colour difference between predicted and reference CIELAB values of ColourChecker (a), Profiling (b) and Testing(c) colour chart in case of ICC based RGB to CIELAB transformation.

\subsection{Polynomial models}

The colour difference between predicted and reference CIELAB values of the process, where ColorChecker was set to be the training set, is presented in Table 4 and Figure 7. The best results are obtained for polynomial model D3 (See table 1 in 2.3) but models D1, D2 and D4 predicted colour of all patches with similar results. The black colour patch of ColorChecker target exhibits the largest colour difference in all D1-D5 models. In case of Profiling and Testing colour 
target worse prediction can be observed especially in darker green and violet colours. Model D5 predicted very poorly the colour representation of light colour patches and saturated orange patches in Profiling and Testing colour chart. The approach applying polynomial models seems to have worse results, than the ICC based transformation.

Table 4: Colour differences in case of polynomial transformations - ColorChecker as training set

\begin{tabular}{|c|r|c|c|c|c|c|}
\hline & & D1 & D2 & D3 & D4 & D5 \\
\hline \multirow{4}{*}{ Median } & ColorChecker & 1.42 & 1.26 & 1.29 & 0.89 & 0.21 \\
\cline { 2 - 7 } & Profiling & 2.34 & 2.17 & 2.11 & 2.37 & 3.61 \\
\cline { 2 - 7 } & Testing & 1.97 & 1.88 & 1.92 & 2.10 & 3.84 \\
\hline \multirow{4}{*}{ Minimum } & ColorChecker & 0.19 & 0.19 & 0.18 & 0.13 & 0.00 \\
\cline { 2 - 7 } & Profiling & 0.80 & 0.48 & 0.56 & 0.35 & 0.69 \\
\cline { 2 - 7 } & Testing & 0.66 & 0.57 & 0.71 & 0.36 & 0.64 \\
\hline \multirow{3}{*}{ Maximum } & ColorChecker & 2.65 & 5.62 & 4.22 & 4.81 & 3.15 \\
\cline { 2 - 7 } & Profiling & 6.66 & 6.74 & 6.74 & 6.78 & 37.87 \\
\cline { 2 - 7 } & Testing & 6.86 & 6.04 & 5.69 & 5.79 & 37.98 \\
\hline
\end{tabular}
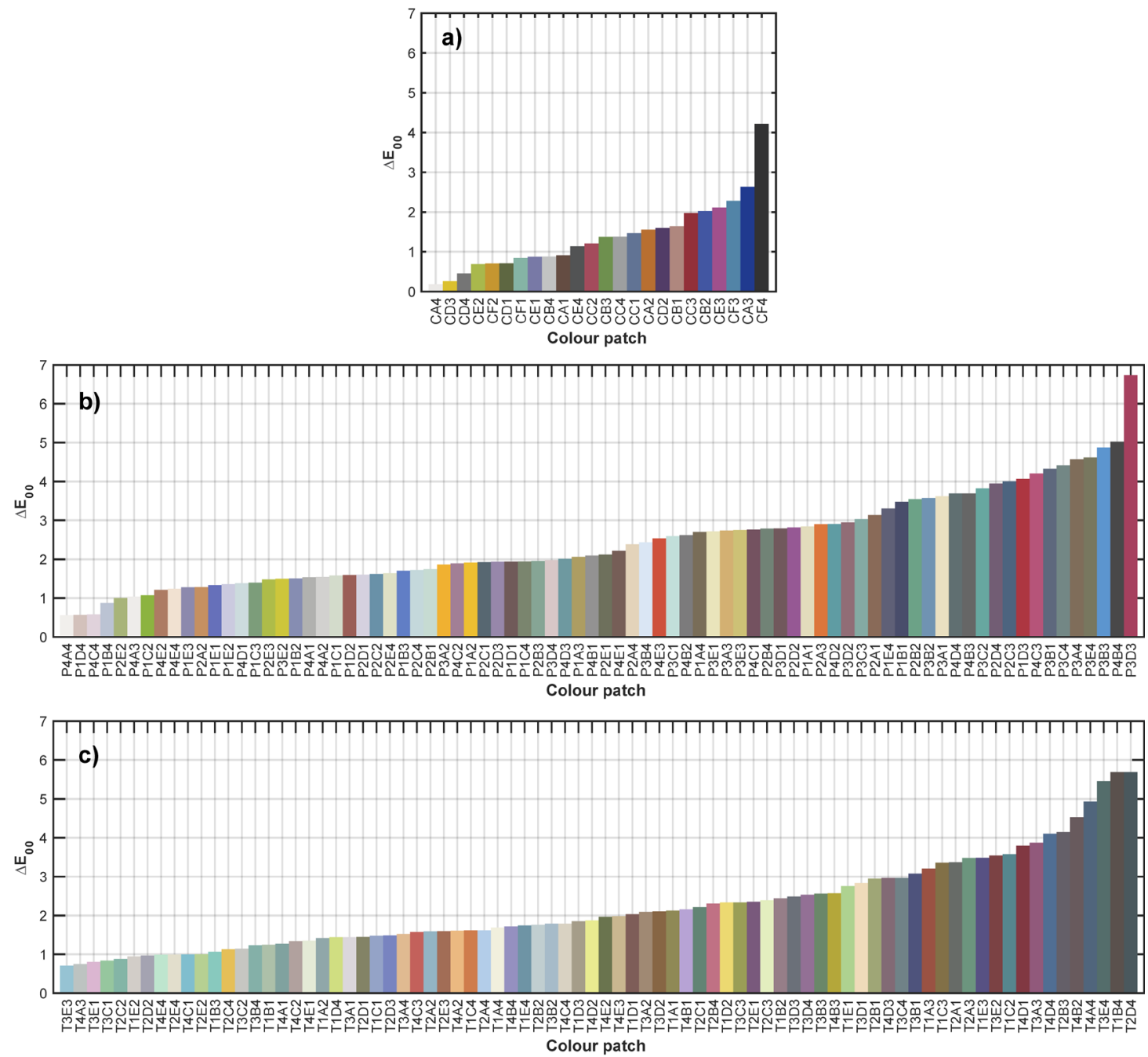

Figure 7: Colour difference between predicted and reference CIELAB values of ColourChecker (a), Profiling (b) and Testing(c) colour chart in case of application of D3 polynomial model utilising ColorChecker as training set.

When the Profiling colour chart was utilised as training, significant improvement can be seen on prediction of CIELAB values of Profiling and Testing charts' colour patches (see Table 5 and Figure 8). 
The $\Delta E_{00}$ does not exceed value 2 for about $75 \%$ of Profiling and Testing colour charts' patches. However, the prediction of CholorChecker patches gets worse especially when D4 model is applied. Again, more complicated models do not dramatically improve the colour prediction. The difference between goodness of prediction between ColorChecker and custom made colour charts is assigned to different diffusive properties of colour patches.

Table 5: Colour differences in case of polynomial transformations - Profiling colour chart as training set

\begin{tabular}{|c|r|c|c|c|c|c|}
\hline & & D1 & D2 & D3 & D4 & D5 \\
\hline \multirow{4}{*}{ Median } & ColorChecker & 2.66 & 2.70 & 2.65 & 2.85 & 2.41 \\
\cline { 2 - 7 } & Profiling & 1.25 & 1.18 & 1.26 & 0.90 & 0.87 \\
\cline { 2 - 7 } & Testing & 1.28 & 1.30 & 1.19 & 1.15 & 1.24 \\
\hline \multirow{3}{*}{ Minimum } & ColorChecker & 0.89 & 1.19 & 1.13 & 0.93 & 0.63 \\
\cline { 2 - 7 } & Profiling & 0.43 & 0.42 & 0.44 & 0.13 & 0.22 \\
\cline { 2 - 7 } & Testing & 0.25 & 0.20 & 0.19 & 0.21 & 0.42 \\
\hline \multirow{3}{*}{ Maximum } & ColorChecker & 4.21 & 7.04 & 7.39 & 11.81 & 6.81 \\
\cline { 2 - 7 } & Profiling & 4.69 & 4.70 & 4.47 & 3.30 & 3.32 \\
\cline { 2 - 7 } & Testing & 5.84 & 3.89 & 3.86 & 3.74 & 6.32 \\
\hline
\end{tabular}
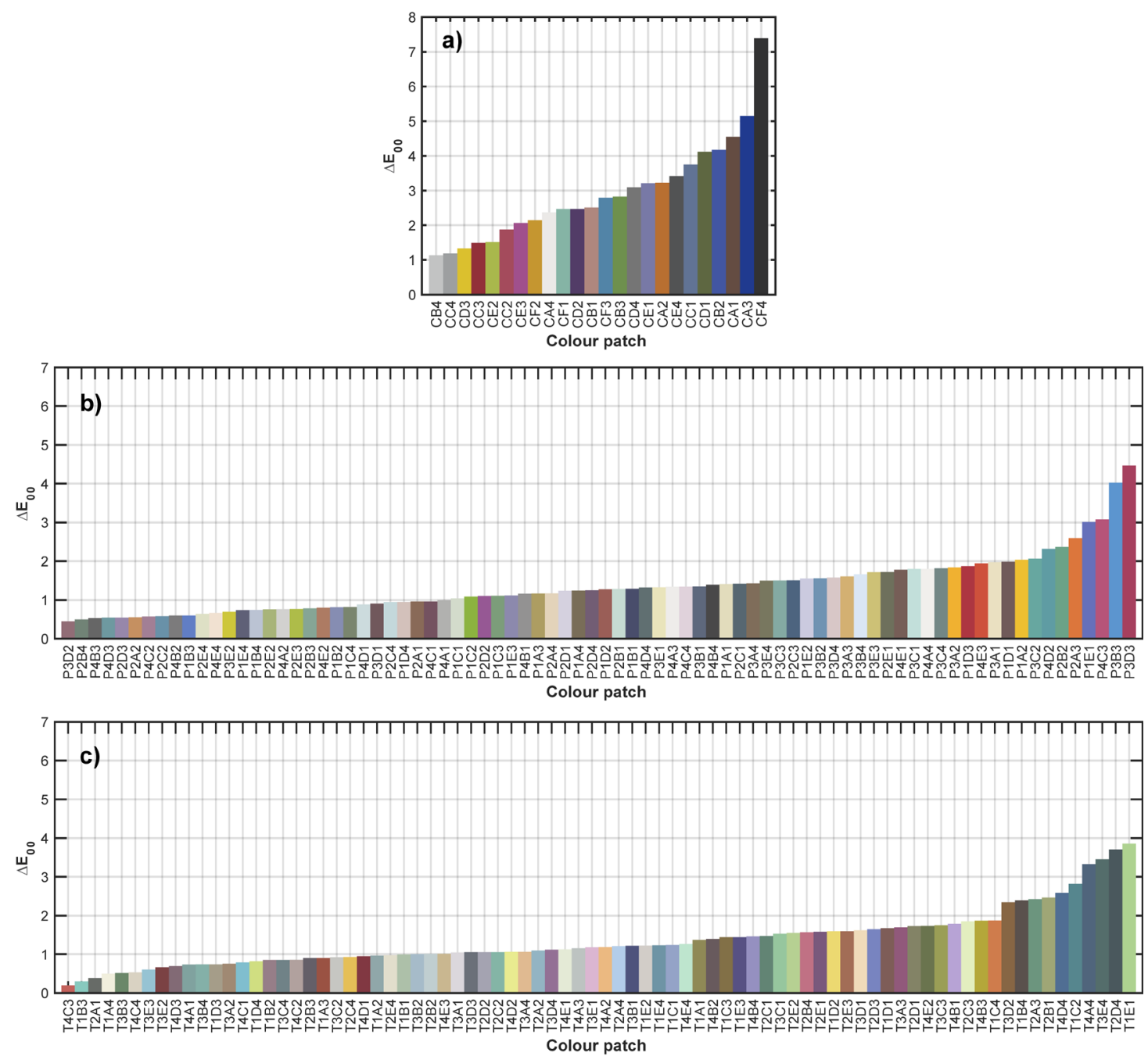

Figure 8: Colour difference between predicted and reference CIELAB values of ColourChecker (a), Profiling (b) and Testing(c) colour chart in case of application of D3 polynomial model utilising Profiling colour chart as training set. 


\section{CONCLUSIONS}

Two approaches of transforming RGB values to CIELAB values were evaluated, one ICC based transformation and 5 transformations using polynomial models of different degrees. Custom made test charts and ColorChecker Passport were utilised in model preparation and evaluation of CIELAB prediction. When the ColorChecker is used as the training set in preparation of ICC profile and also determination of polynomial models, the ICC based transformation performs slightly better. When the custom made training set was applied, the prediction was better only in case of colour patches of the same surface properties. Obtained results show, that more complicated polynomial models do not have serious impact on the goodness of CIELAB prediction.

\section{REFERENCES}

[1] Abdullah, N., Talib, A.R.A., Jaafar, A.A., Salleh, M.A.M., Chong, W.T.: "The basics and issues of thermochromic liquid crystal calibrations", Experimental Thermal and Fluid Science, 34(8), 10891121, 2010. doi: 10.1016/j.expthermflusci.2010.03.011

[2] Bianco, S., Schnettini, R., Vanneschi, L.: "Empirical modeling for colorimetric characterization of digital cameras", International Conference on Image Processing, 16 ${ }^{\text {th }}, 2009$, 3469-3472, 2009. doi: 10.1109/ICIP.2009.5413828

[3] Bourque, A.N., White, M.A.: "Control of thermochromic behaviour in crystal violet lactone (CVL)/alkyl gallate/alcohol ternary mixtures", Canadian Journal of Chemistry, 93, 22-31, 2015. doi: 10.1139/cjc-2014-0251

[4] Cheung, V., Hardeberg, C. Li, J., Connah, D., Westland, S.: "Characterization of trichromatic color cameras by using a new multispectral imaging technique", Journal of the Optical Society of America A 22(7), 1231-1240, 2005. doi: 10.1364/JOSAA.22.001231

[5] Cheung, V., Westland, S., Connah D., Ripamonti, C: "A comparative study of the characterisation of colour cameras by means of neural networks and polynomial transforms", Coloration Technology, 120, 19-25, 2004. doi: 10.1111/j.1478-4408.2004.tb00201.x

[6] Cukurel, B., Selcan, C., Arts, T.: "Color theory perception of steady wide band liquid crystal thermometry", Experimental Thermal and Fluid Science, 39, 112-122, 2012. doi: 10.1016/j.expthermflusci.2012.01.015

[7] Falcon Eyes, "DVR-630D/630DVC", Falconeyes, URL: http://www.falconeyes.com.hk/Product.aspx?id=1512 (last request: 2018-03-02).

[8] Farina, D.J., Hacker, J.M., Moffat, R.J., Eaton, J.K.: "Illuminant invariant calibration of thermochromic liquid crystals", Experimental Thermal and Fluid Science, 9, 1-12, 1994. doi: 10.1016/08941777(94)90002-7

[9] Finlayson, G. D., Mackiewicz, M., Hulbert, A.: "Color Correction Using Root-Polynomial Regression", IEEE Transactions on Image Processing, 24(5), 1460-1470, 2015. doi: 10.1109/TIP.2015.2405336

[10] Hong, G., Rhodes, P. A., Luo, M. R.: "A study of digital camera colorimetric characterization based on polynomial modelling", Color Research \& Application, 26, 76-84, 2001. doi: 10.1002/15206378(200102)26:1<76::AID-COL8>3.0.CO;2-3

[11] Jiang, J., Liu, D., Gu, Jinwei., Susstrunk, S.: "What is the space of spectral sensitivity functions for digital color cameras?", Applications of Computer Vision (WACV), (IEEE, 2013), 168-179. doi: 10.1109/WACV.2013.6475015

[12] Johnson T.: "Methods for characterizing colour scanners and digital cameras", Displays 16(4), 183-191, 1996. doi: 10.1016/0141-9382(96)01012-8

[13] Panák, O., Držková M., Kailová N., Syrový T.: "Colorimetric analysis of thermochromic samples in different forms employing a digital camera", Measurement 127, 554-64, 2018. doi: 10.1016/j.measurement.2018.06.025

[14] Smith, C.R., Sabatino, D.R., Praisner, T.J.: "Temperature sensing with thermochromic liquid crystals", Experiments in Fluids, 30(2), 190-201, 2001. doi: 10.1007/s003480000154

[15] Sole, A., Farup, I., Tominaga, S.: "Image based reflectance measurement based on camera spectral sensitivities", El - Measuring, Modeling, and Reproducing Material Appearance, 2470-1173, 2016. doi: 10.2352/ISSN.2470-1173.2016.9.MMRMA-360

[16] Vejrazka, J., Marty, Ph.: "An alternative technique for the interpretation of temperature measurements using thermochromic liquid crystals", Heat Transfer Engineering, 28(2), 154-16, 2007. doi: 10.1080/01457630601023641 
[17] Westland, S., Ripamonti, C. Cheung, V.: “Computational colour science using MATLAB", $2^{\text {nd }}$ ed, (John Wiley \& Sons Ltd., Chichester, 2012).

[18] X-Rite, "ColorChecker Targets", Xritephoto, URL:

http://xritephoto.com/ph_product_overview.aspx?ID=1192\&

ction=Support\&SupportID=5884\&catid=28 (last request: $2018-09-20)$.

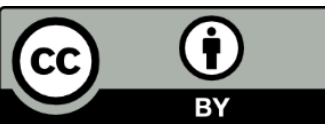

(C) 2018 Authors. Published by the University of Novi Sad, Faculty of Technical Sciences, Department of Graphic Engineering and Design. This article is an open access article distributed under the terms and conditions of the Creative Commons Attribution license 3.0 Serbia

(http://creativecommons.org/licenses/by/3.0/rs/). 